\title{
CORRIGENDUM
}

\section{Transcription factor plasmid binding modulates microtubule interactions and intracellular trafficking during gene transfer}

\author{
MA Badding ${ }^{1,3}$, EE Vaughan ${ }^{2,3}$ and DA Dean ${ }^{1,2}$ \\ ${ }^{1}$ Division of Neonatology, Department of Pediatrics, School of Medicine and Dentistry, University of Rochester, Rochester, NY, \\ USA and ${ }^{2}$ Division of Pulmonary and Critical Care Medicine, Northwestern University, Chicago, IL, USA
}

Correspondence: Dr DA Dean, Division of Neonatology, Department of Pediatrics, School of Medicine and Dentistry, University of Rochester, Box 850, 601 Elmwood Avenue, Rochester, NY 14642, USA. E-mail: david_dean@urmc.rochester.edu

${ }^{3}$ These authors are co-first authors.

Gene Therapy (2012) 19, 354; doi:10.1038/gt.2011.113; published online 21 July 2011

Correction to: Gene Therapy (2012) 19, 338-346; doi:10.1038/ The authors would like to apologize for this error. gt2011.96; published online 30 June 2011

Since the online publication of this paper, the authors have noticed that the first two authors were not listed as co-first authors. The correct details are shown above. 